\title{
UPPER ATRIO-VENTRICULAR NODAL BEATS PRECIPITATED BY VENTRICULAR EXTRASYSTOLES WITH RETROGRADE CONDUCTION
}

\author{
BY \\ A. SCHOTT \\ From Queen Mary's Hospital for the East End, London \\ Received November 17, 1954
}

The arrhythmias observed in the cases to be described show combinations of rare features which to our knowledge have not hither to been recorded.

\section{CASE 1}

The patient was a 75-year-old man with a four years' history of nocturnal attacks of cardiac asthma, increasing dyspnœa, and œdema of the feet. On admission he was found to be suffering from hypertensive cardiac failure. The blood pressure was $210 / 120 \mathrm{~mm}$. $\mathrm{Hg}$. The heart action was regular, rate 112. Radiological examination showed enlargement of the heart with evidence of pulmonary congestion. Prior to admission the patient had not received any medication. In the course of three-and-a-half weeks stay in hospital treatment included digoxin, $0.25 \mathrm{mg}$. twice daily, mersalyl injections, $2 \mathrm{ml}$. twice weekly, and aminophylline, 3 grains thrice daily. His condition improved considerably.

A routine electrocardiogram taken three days after admission (Fig. 1) showed sinus rhythm with a fairly constant cycle length of $0.76 \mathrm{sec}$. (rate 78), P-R intervals of $0.16 \mathrm{sec}$., left ventricular strain, and ventricular extrasystoles. Their shape varied somewhat owing to the effect of digitalis, and to distortion of $P$ waves (see below). Their coupling varied between 0.42 and $0.68 \mathrm{sec}$. Some of the extrasystoles showed a sharply inverted $\mathbf{P}^{\prime}$ wave in their final deflection in leads II and III, indicating retrograde conduction to the auricles.* The $\mathbf{P}$ waves of the first post-extrasystolic beats after such extrasystoles were sharply inverted in leads II and III and the P-R intervals measured only $0.12 \mathrm{sec}$., whereas the $\mathbf{P}$ waves of the post-extrasystolic beats after ventricular extrasystoles without retrograde conduction were identical with those of other sinus beats. (The P wave of the last post-extrasystolic beat in lead III was actually a fusion beat between a sinus $P$ wave due at the time and a sharply inverted $P$ wave; this could be ascertained from later records, see Fig. 2.)

In view of the great variations in the coupling of the extrasystoles, parasystole was suspected, but in longer records taken four days later, which showed even greater variations in coupling, a parasystolic mechanism could not be established. Instead, gradually decreasing couplings were found, whereby those extrasystoles falling comparatively early in diastole showed retrograde conduction to the auricles and were followed by a post-extrasystolic beat with inverted $\mathbf{P}$ waves in leads II and III and shortened P-R intervals.

Thus lead II (Fig. 2) showed at its beginning two sinus beats occurring at an interval of $0.86 \mathrm{sec}$. The following beat was a ventricular extrasystole with the long coupling of $0.74 \mathrm{sec}$., recorded at a time when most of the $\mathbf{P}$ wave of the next sinus beat had already been inscribed. This extrasystole did not show any features suggestive of retrograde conduction, and the following beat had a normal

: P waves, that are sharply inverted in leads II and III and are indicative of retrograde conduction of an extrasystole, are called $\mathbf{P}^{\prime}$ waves in this paper. 


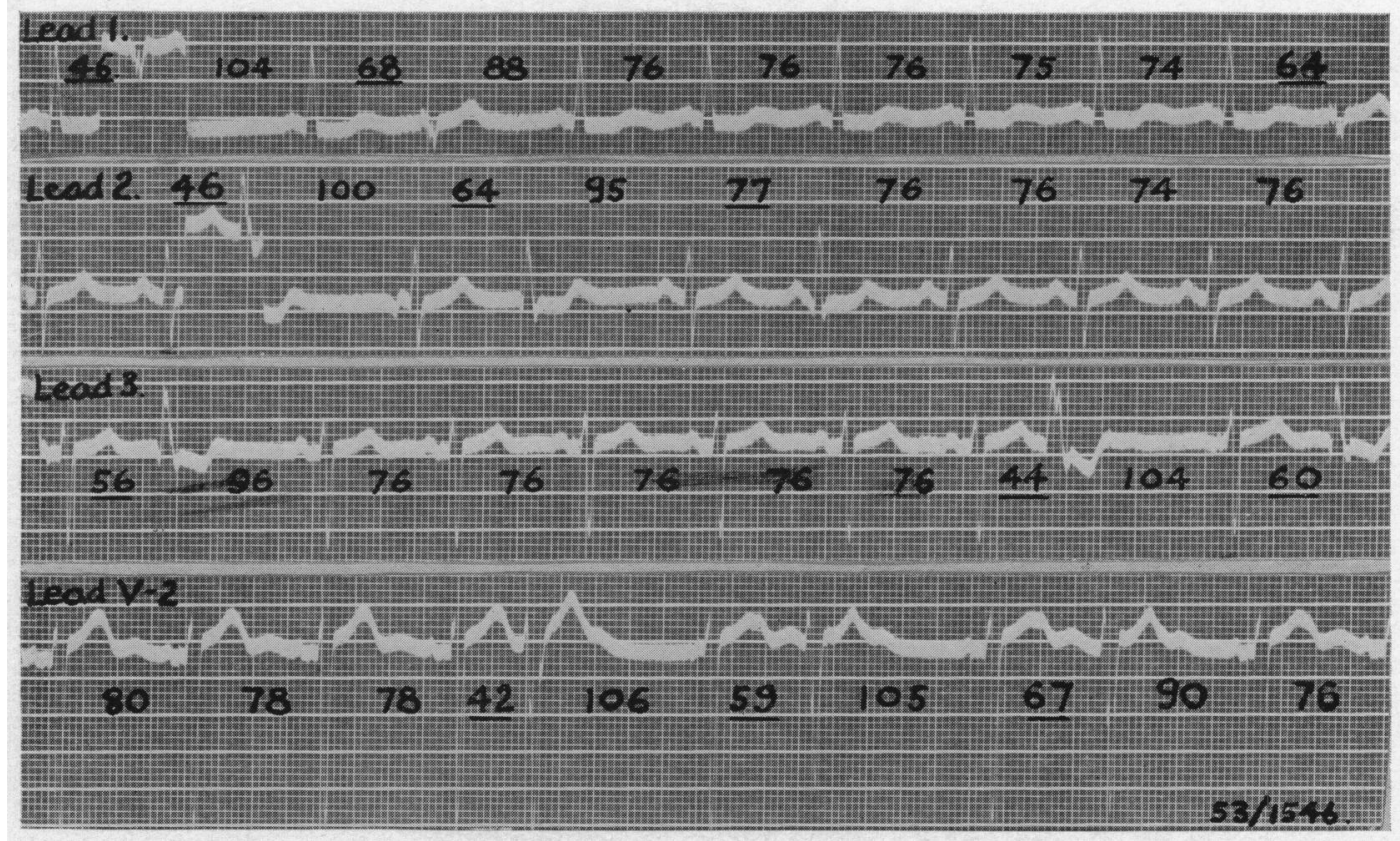

Fig. 1.-For explanation, see text. In this and the subsequent figures numbers indicate intervals in sec./100. Couplings of extrasystoles are underlined.

$\mathrm{P}$ wave, preceding the QRS complex at a $\mathrm{P}-\mathrm{R}$ interval of $0.16 \mathrm{sec}$. The next beat was a ventricular extrasystole of essentially the same shape as the preceding one, except for some variation early in its final deflection produced by the $\mathrm{P}$ wave of the next sinus beat due at that time, but its coupling was only $0.65 \mathrm{sec}$. The first post-extrasystolic beat was a normal sinus beat. The post-extrasystolic intervals after these two extrasystoles were compensatory. The next extrasystole followed with the still shorter coupling of $0.56 \mathrm{sec}$; ; at the foot of the descending limb of its $R$ wave a $\mathbf{P}^{\prime}$ wave is visible, strongly suggestive of retrograde conduction. The first post-extrasystolic beat had a sharply inverted $\mathbf{P}$ wave, its $\mathbf{P}-\mathbf{R}$ interval being shortened to $0.11 \mathrm{sec}$. Similar features were observed in the post-extrasystolic beat following the next extrasystole, whose coupling was only $0.40 \mathrm{sec}$. and which had also a $\mathbf{P}^{\prime}$ wave in its final deflection. The post-extrasystolic intervals after these last two extrasystoles were shorter than compensatory, the first only slightly, but the second falling short by about $0 \cdot 15 \mathrm{sec}$. It is also significant that the interval between the $\mathrm{P}^{\prime}$ wave of the extrasystole and the following inverted $P$ wave equals the cycle length of the sinus rhythm, namely $0.84 \mathrm{sec}$. As the first post-extrasystolic beats following, respectively, the last two extrasystoles have all the characteristics of upper atrio-ventricular nodal beats, it has to be concluded that the extrasystoles with retrograde conduction, and only these, precipitated a transient A-V rhythm. In lead II, this consisted of only one beat. The next beat (the second beat of the lower strip of lead II) showed a mainly upright $\mathbf{P}$ wave with a very small initial downward deflection. It differed in shape from the sinus $P$ waves and its $P-R$ interval was shortened to 0.11 sec., while the subsequent beats were normal sinus beats with $\mathrm{P}-\mathrm{R}$ intervals of $0.16 \mathrm{sec}$. The $\mathrm{P}$ wave of this second post-extrasystolic beat has to be considered a fusion beat, intermediate in shape between the rounded upright sinus $P$ waves and the sharply inverted $P$ waves of upper A-V nodal beats.

Similar conditions were recorded in lead III. There were again four ventricular extrasystoles with decreasing coupling. The first two occurred after the longer couplings of 0.76 and $0.70 \mathrm{sec}$., respectively, did not show any features suggestive of retrograde conduction, and were followed after a compensatory pause by post-extrasystolic beats which were sinus beats with normal $\mathbf{P}$ waves and 


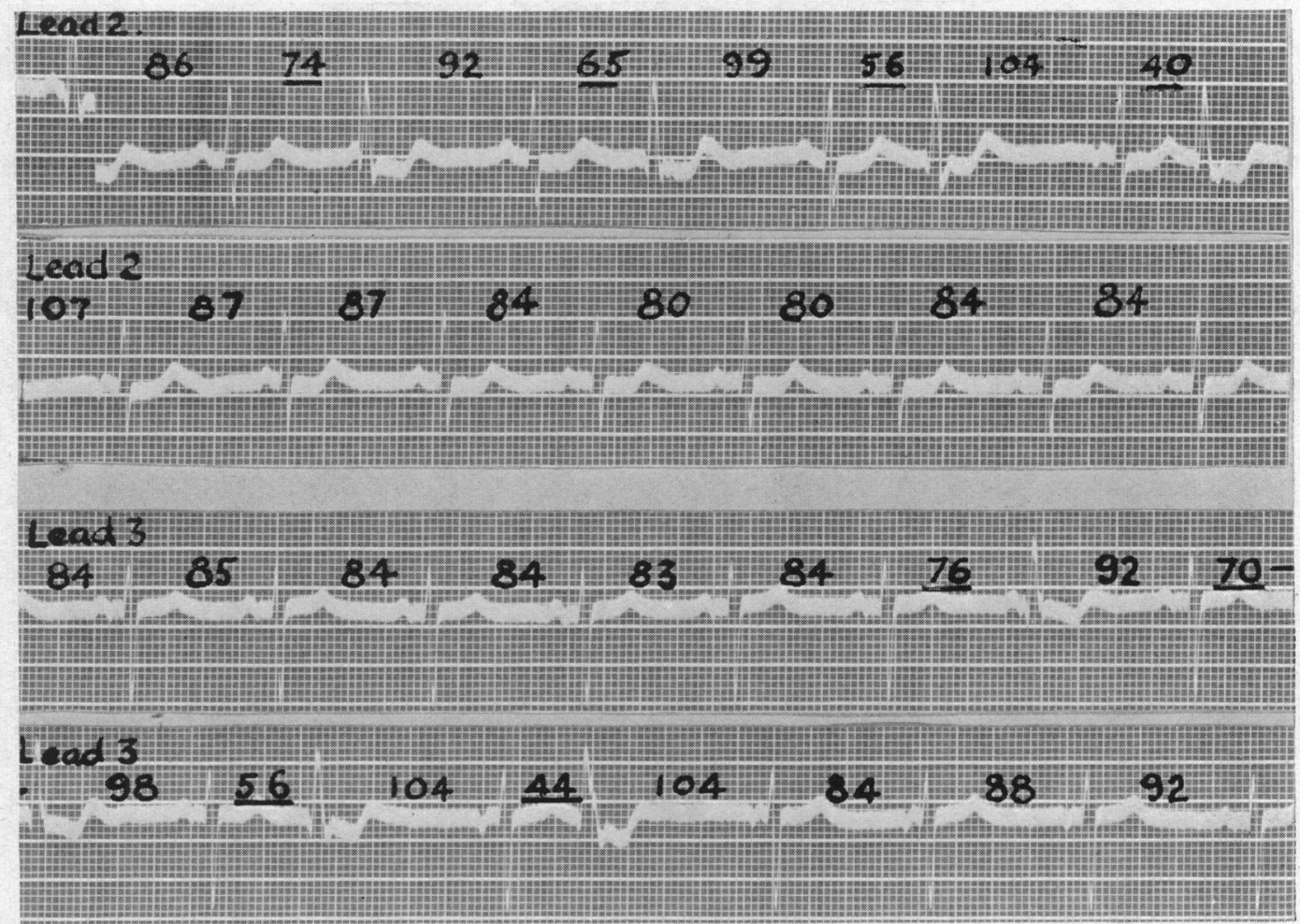

FIG. 2.-Recorded four days after Fig. 1. The two strips of leads II and III are continuous. Note gradually decreasing coupling of consecutive extrasystoles, retrograde conduction of extrasystoles falling early in diastole, and upper A-V nodal post-extrasystolic beats after these only. For further explanation, see text.

normal P-R intervals. The last two extrasystoles occurred with the shorter couplings of 0.56 and 0.44 sec., respectively, had $\mathrm{P}^{\prime}$ waves in their final deflections, and were followed, after an interval shorter than compensatory, by beats with sharply inverted $\mathbf{P}$ waves and shortened $\mathbf{P}-\mathbf{R}$ intervals. The most noteworthy feature of this lead is that the last extrasystole with retrograde conduction was followed, not by one, but by three upper A-V nodal beats; subsequently sinus rhythm became re-established. The cycle lengths of the A-V nodal beats, measuring 0.84 and $0.88 \mathrm{sec}$., were very close to that of the sinus rhythm. Similar features were recorded in V4 (not shown). In this lead, one group of bigeminal rhythm was initiated by a fusion beat, with a ventricular complex intermediate in shape between that of the sinus beats and the extrasystoles, which had the unusually long coupling of $0.80 \mathrm{sec}$. The $\mathrm{P}^{\prime}$ waves were not distinct in any of the chest leads.

A further record (Fig. 3), taken six days after the preceding one and reproducing lead II and a longer strip of V1, showed that the arrhythmia had altered in several important respects. The rate of the sinus rhythm was unchanged, the cycle length being $0 \cdot 80-0.84 \mathrm{sec}$. The two extrasystoles in lead II were recorded in a long period of undisturbed sinus rhythm. They had the comparatively short coupling of $0.44 \mathrm{sec}$., showed distinct $\mathrm{P}^{\prime}$ waves in their final deflections, but were followed after a compensatory post-extrasystolic interval by normal sinus beats. Contrary to the condition found six days earlier (Fig. 2), these beats did not precipitate A-V nodal beats. The time relations suggest that the retrograde impulse did not reach the S-A node, the dominant sinus rhythm thus remaining undisturbed.

In lead V1, altogether sixteen extrasystoles of the same shape were recorded, divided into two groups comprising five and eleven extrasystoles respectively, the two groups being separated by three 


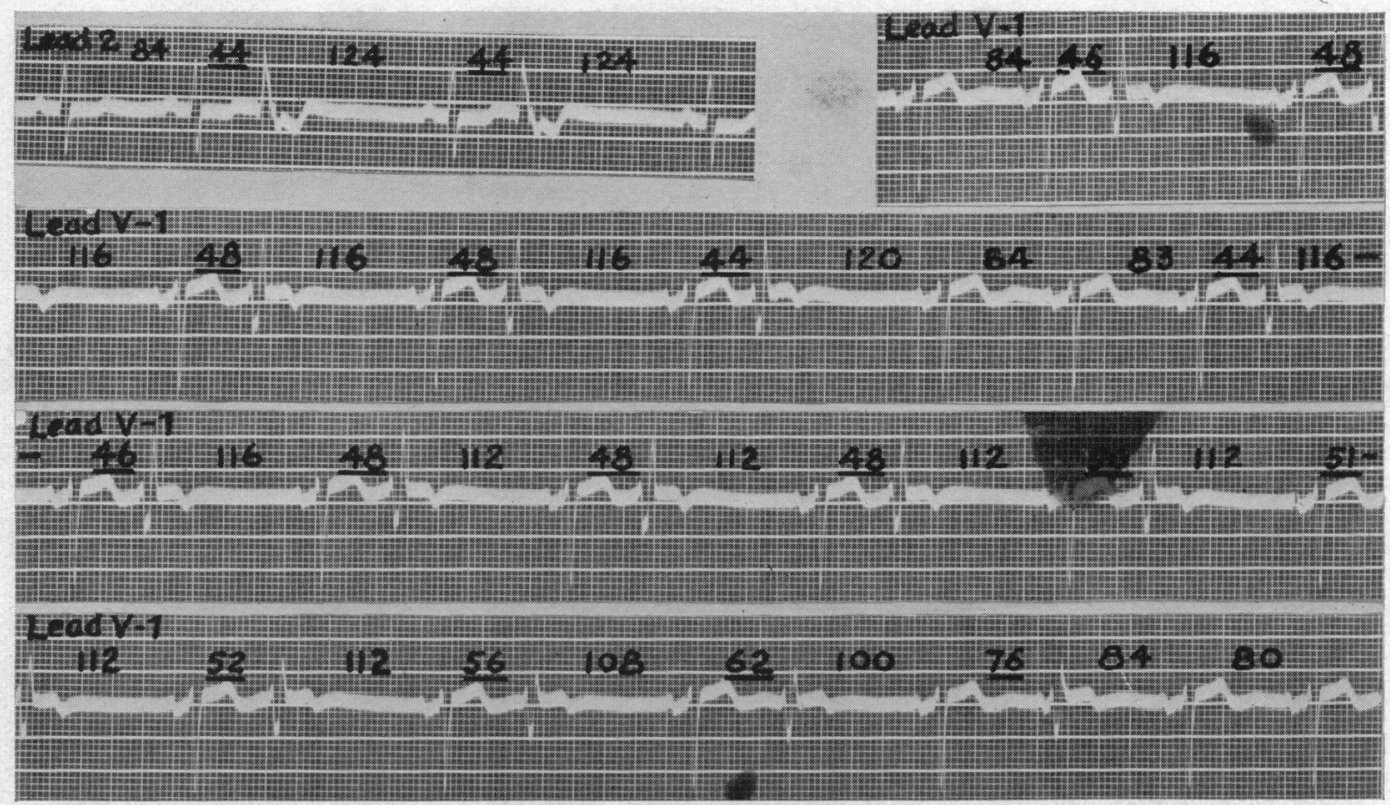

Fig. 3.--Recorded six days after Fig. 2. All strips of lead V1 are continuous. Lead II: Two extrasystoles with retrograde conduction followed after a compensatory pause by sinus beats. Lead V1: Practically constant coupling of the five extrasystoles of the first group, gradually increasing coupling in the second group of eleven extrasystoles.

successive sinus beats. The extrasystoles of the first group had couplings of $0.46,0.48,0.48,0.48$, $0.44 \mathrm{sec}$., i.e. varying only within such narrow limits that the coupling could be considered as constant. In the second group, the couplings of the extrasystoles gradually increased from 0.44 to $0.76 \mathrm{sec}$., the post-extrasystolic intervals being compensatory; subsequently sinus rhythm prevailed. Whether these extrasystoles were reversedly conducted to the auricles cannot be deduced from the tracing.

\section{Discussion}

The records of this patient showed several unusual features which warrant some more detailed discussion, namely, (1) systematic variations in the coupling of the extrasystoles; (2) fairly accurate coupling on one occasion; (3) retrograde conduction of some of the ventricular extrasystoles; and (4) transient upper A-V nodal rhythm following only ventricular extrasystoles with retrograde conduction.

Variations in Coupling. A parasystolic mechanism could not be established. Instead, systematic changes in the lengths of coupling were found. In the first record, the couplings of consecutive extrasystoles increased before undisturbed sinus rhythm became re-established. In lead V2 (Fig. 1), this increase ranged from 0.42 to $0.67 \mathrm{sec}$.; in lead V4 (not shown), from 0.44 to 0.62 , and in a subsequent bigeminal group from 0.48 to $0.64 \mathrm{sec}$. It is important to note that these changes in coupling occurred without any significant alteration in the sinus rate. This condition is uncommon. Examples have been described by Zander (1927), Mack and Langendorf (1950) (Case 1), Scherf and Schott (1953) (Fig. 130), and also form part of an observation by Goldenberg and Scherf (1928). Zander, and Mack and Langendorf believe that disturbances of conduction were responsible for the gradually increasing coupling. Goldenberg and Scherf, however, found this explanation unacceptable in their case and gave several reasons for this view. In the first place, they rejected Zander's assumption that retrograde conduction to the ectopic focus through the peripheral branch of the Purkinje system was completely blocked and the focus thus accessible only by delayed conduction in the orthograde direction; this is not consistent with experimental findings demonstrating that such retro- 
grade conduction is preserved even in the presence of pronounced myocardial damage with grave impairment of orthograde conduction.

Furthermore, the long couplings in Goldenberg and Scherf's case, sometimes exceeding $0.80 \mathrm{sec}$., were cited by these authors as an argument against delay of conduction being the responsible factor. The same holds good for the present case. Couplings of $0.76 \mathrm{sec}$. were found twice, one of 0.77 , and on one occasion one of $0.8 \mathrm{sec}$., producing a fusion beat, was recorded.

The most cogent reason is, however, that - as in Goldenberg and Scherf's case-the reverse phenomenon was also observed, namely, a gradual decrease in the coupling of consecutive extrasystoles. Thus, in lead II (Fig. 2), the couplings gradually decreased from 0.74 to $0.40 \mathrm{sec}$., in lead III from 0.76 to 0.44 , and in V4 (not shown) from 0.76 to 0.40 and from 0.80 to $0.40 \mathrm{sec}$. in two separate groups, respectively. In the great majority of cases, the effect upon conduction of successive impulses traversing the same path consists in progressive delay in conduction. The reverse is only observed in comparatively rare exceptions (some instances of successive auricular extrasystoles or of paroxysmal tachycardia). For these reasons Goldenberg and Scherf (1928) assumed that, in their case, some disturbance of impulse formation was the underlying factor. This seems also the most acceptable explanation for the present case, as far as the variations in length of coupling are concerned.

Temporary Accurate Coupling. On one occasion (Fig. 3, lead V1), a group consisting of five successive bigeminal groups was recorded, with couplings of the extrasystoles of $0 \cdot 46,0.48,0 \cdot 48,0 \cdot 48$, and $0.44 \mathrm{sec}$. This could be considered as within the range of accurate coupling. While not too much importance should be attached to this short period, it may be mentioned that accurate coupling at some time was also observed in most of the few comparable cases already quoted (Zander, 1927; Goldenberg and Scherf, 1928; Mack and Langendorf, 1950). If in the present case this short period is accepted as indicating temporary accurate coupling, this observation would be a further instance of the transition in the same case from extrasystoles with systematically varying coupling to those of the common variety with constant coupling. The change from ectopic beats with varying coupling to extrasystoles in the strict sense of the term, i.e. with accurate coupling, is altogether rare, even if the cases of transition from parasystole to extrasystoles are included (Scherf and Schott, 1930; Rothberger, 1931; Faltitschek and Scherf, 1932; Vedoya and Battini, 1939).

Retrograde Conduction of some of the Ventricular Extrasystoles. Retrograde conduction to the auricles of ventricular extrasystoles is indicated by a $\mathbf{P}^{\prime}$ wave, which is sharply inverted in leads II and III, following the QRS complex of the extrasystole at an interval compatible with retrograde conduction. The diagnosis is rendered more certain if extrasystoles without retrograde conduction are recorded in the same lead, so that their shape undistorted by $\mathbf{P}^{\prime}$ waves is available for comparison, and if the post-extrasystolic interval is not compensatory. Both these conditions prevail in the present case; and it can be shown that only those extrasystoles were reversedly conducted which occurred comparatively early in diastole, i.e. with shorter couplings. Thus, in leads II and III of Fig. 1, only two extrasystoles with couplings of 0.44 and $0.46 \mathrm{sec}$. showed retrograde conduction, whereas others with couplings ranging from 0.56 to $0.77 \mathrm{sec}$. did not demonstrate this phenomenon. Similarly, in leads II and III of Fig. 2, four extrasystoles with couplings ranging from 0.40 to $0.56 \mathrm{sec}$. were conducted to the auricles, whereas another four with couplings between 0.65 and $0.76 \mathrm{sec}$. were not.

A similar observation was made by Kistin and Landowne (1951), who established by means of cesophageal leads that the retrograde conduction of ventricular extrasystoles in man is far commoner than usually assumed. These authors point out that extrasystoles falling late in diastole tend to occur immediately before or after the $\mathbf{P}$ wave of the next sinus beat, that is, at a time when activation of the auricles by the next S-A impulse has started, which precludes retrograde activation. It is thus the extrasystoles with the shorter couplings which are most likely to show retrograde conduction.

Another possible factor may be the supernormal phase, namely the assumption that retrograde conduction takes place during the supernormal phase of conductivity of the preceding beat. This mechanism was suggested to account for the retrograde conduction in parasystole of ectopic beats with a comparatively short coupling to the preceding beat (Scherf and Schott, 1953). The order of length of the supernormal phase, which was found in some of the few cases in which its presence could be accepted as established in the human heart (e.g. Jervell, 1934; Froment et al., 1939), is consistent with this assumption.

Transient Upper A-V Nodal Rhythm following only Ventricular Extrasystoles with Retrograde Conduction. In the standard leads, uncomplicated upper A-V nodal rhythm is characterized by ventricular complexes of 
supra-ventricular shape, preceded at an abnormally short $\mathbf{P}-\mathbf{R}$ interval by $\mathbf{P}$ waves which are low positive or iso-electric in lead I and sharply inverted in leads II and III. In some of the chest leads such P waves are also inverted. Post-extrasystolic beats following ventricular extrasystoles with retrograde conduction to the auricles and showing these characteristics are seen in Fig. 1 after the first extrasystole in leads II and V2, and in Fig. 2 after the third and fourth extrasystole in leads II and III. The post-extrasystolic intervals terminated by upper A-V nodal beats were shorter than compensatory. The interval between the $\mathbf{P}^{\prime}$ wave of the extrasystole and the inverted $\mathbf{P}$ wave of the first post-extrasystolic A-V nodal beat equalled, or was slightly $(0.04 \mathrm{sec}$.) longer than the cycle length of the prevailing sinus rhythm. These time relations suggest that the retrograde conduction extended to the sinus node. A-V nodal post-extrasystolic beats were not observed after extrasystoles without retrograde conduction.

The post-extrasystolic intervals after extrasystoles with retrograde conduction being long, their termination by escape beats can be easily understood. The unusual feature about these beats is that they showed the characteristics of upper A-V nodal beats, with preceding activation of the auricles. In man, nodal escape beats are nearly always of the variety with simultaneous activation of auricles and ventricles. In the latter, $\mathbf{P}$ waves are either absent or, being buried in the ventricular complex, are recognizable only by some slight deformation of the QRS complex. It was emphasized by Scherf (1931) that in A-V nodal escape beats a P wave never precedes the ventricular complex. In the present case, transient upper A-V nodal rhythm extended at times over several beats. Thus, in Fig. 2, the last extrasystole in lead III was followed by three upper A-V nodal beats; and in lead II the second post-extrasystolic beat following the last extrasystole had a fusion $P$ wave, intermediate in shape between the $P$ waves of the sinus and the A-V nodal beats, and a shortened P-R interval.

We have been unable to find a comparable instance in previous work on the arrhythmias. The only case known to us, which shows this phenomenon, is the following unpublished observation of Scherf which he has kindly placed at our disposal to be included in this paper.

\section{CASE 2}

The tracing (Fig. 4), recorded in a 63-year-old woman admitted to hospital for gall stones without any cardiac or other complications, demonstrated sinus rhythm with a cycle length of 1.04 sec. (rate 57), and ventricular extrasystoles which, in leads II and III, showed the characteristic $\mathbf{P}^{\prime}$ waves in the final deflections, indicating retrograde conduction. The first post-extrasystolic beat, following an interval far shorter than compensatory, had a flat diphasic $\mathbf{P}$ wave in lead II and a frankly inverted $\mathbf{P}$ wave in lead III. The $\mathbf{P}-\mathbf{R}$ intervals of these post-extrasystolic beats were shortened to $0.08 \mathrm{sec}$. in lead II and $0.1 \mathrm{sec}$. in lead III, compared with $\mathrm{P}-\mathrm{R}$ intervals of 0.12 and $0 \cdot 12-0.14 \mathrm{sec}$. of the sinus beats in these two leads. The $\mathbf{P}^{\prime}-\mathbf{P}$ intervals measured $1.14 \mathrm{sec}$, that is, they exceeded the P-P intervals of the sinus rhythm by $0.1 \mathrm{sec}$. Although in the two chest leads $\mathbf{P}^{\prime}$ waves were not recognizable and the changes in the $P$ waves of the post-extrasystolic beats were only very slight, the same conditions can be assumed, since the time relations (lengths of couplings and post-extrasystolic intervals) were identical. This is, therefore, a further instance of ventricular extrasystoles with retrograde conduction, in which the post-extrasystolic intervals were terminated by upper A-V nodal beats. No digitalis had been administered.

\section{Mode of Origin of Transient Upper A-V Nodal Rhythm}

The two observations described demonstrate the temporary precipitation of an upper A-V nodal rhythm by retrograde impulses, and by these only. In order to explain the preceding activation of the auricles, the assumption of any delay in conduction in the ventricles of the A-V nodal beats is not justified, since no evidence of disturbances of intra-ventricular conduction was present in the sinus beats. Moreover, the longer post-extrasystolic intervals created if anything better conditions for conduction.

The phenomenon can best be understood by assuming that the retrograde impulse of the ventricular extrasystole facilitated the conduction of the A-V nodal impulse in the auricles. Therefore, the interval between impulse formation in the A-V node and activation of the auricles was shorter than in the common variety of $\mathrm{A}-\mathrm{V}$ nodal escape beats.

Experimentally, the importance of facilitation in retrograde conduction was established in dogs 


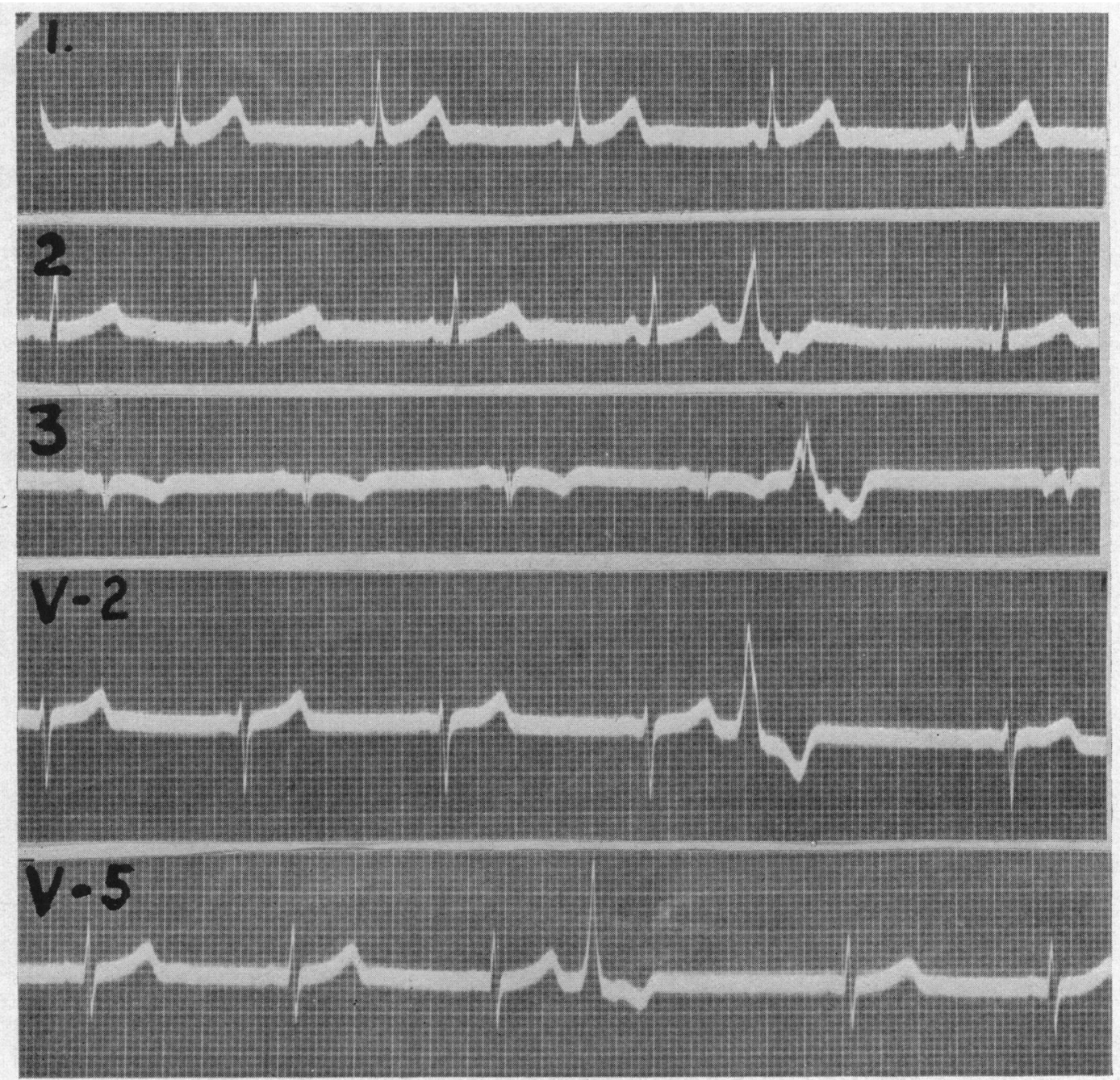

FIG. 4.-Ventricular extrasystoles with retrograde conduction, termination of post-extrasystolic intervals by upper A-V nodal beats. From another patient. Reproduced by courtesy of Dr. D. Scherf.

by Lewis and Oppenheimer (1911). They found that, if a ventricular rhythm was produced by means of induction shocks, the auricles responded to the ventricular beats only after a variable number of cycles. Clinically, this phenomenon has been shown in an observation by Allan (1926). During attacks of paroxysmal ventricular tachycardia, retrograde conduction to the auricles of every second beat became established only after several beats without such retrograde conduction had first occurred at the onset of these attacks.

\section{SUMMARY}

An unusual ectopic arrhythmia is described which showed ventricular extrasystoles with systematically increasing and at other times systematically decreasing coupling. Extrasystoles falling comparatively early in diastole showed signs of retrograde conduction to the auricles and only these were followed by upper A-V nodal post-extrasystolic beats. 
In a second case the post-extrasystolic intervals after ventricular extrasystoles with retrograde conduction to the auricles were also terminated by upper A-V nodal beats.

Reasons are given for the view that the variations in coupling were due to disturbances in impulse formation and not to disturbances in impulse conduction.

Facilitation of conduction in the auricles of the A-V nodal post-extrasystolic beats is put forward as a tentative explanation for the occurrence of A-V nodal escape beats and temporary postextrasystolic A-V nodal rhythm with preceding activation of the auricles.

\section{REFERENCES}

Allan, G. A. (1926). Glas. med. J., 105, 440.

Faltitschek, F., and Scherf, D. (1932). Wien. Arch. inn. Med., 23, 269.

Froment, R., Masson, R., and Gonin, A. (1939). Arch. Mal. Coeur, 32, 849.

Goldenberg, M., and Scherf, D. (1928). Wien. Arch. inn. Med., 15, 257.

Jervell, A. (1934). Acta med. scand., Suppl., 59, p. 626.

Kistin, A. D., and Landowne, M. (1951). Circulation, 3, 738.

Lewis, T., and Oppenheimer, B. S. (1911). Quart. J. Med., 4, 145.

Mack, I., and Langendorf, R. (1950). Circulation, 1, 910.

Rothberger, C. J. (1931). Ergebn. Physiol., 32, 472. P. 675.

Scherf, D. (1931). Wien. klin. Wschr., No. 37.

, and Schott, A. (1930). Klin. Wschr., 9, 2191.

- 1 (1953). Extrasystoles and Allied Arrhythmias. Heinemann, London.

Vedoya, R., and Rodriguez Battini, A. (1939). Rev. argent. Cardiol., 6, 313.

Zander, E. (1927). Acta med. scand., 66, 189. 